\title{
All-optical control of surface plasmons by second-harmonic generation
}

\author{
Sergio G. Rodrigo* \\ Instituto de Ciencia de Materiales de Aragón and Departamento de Física de la Materia Condensada, \\ CSIC-Universidad de Zaragoza, E-50009 Zaragoza, Spain \\ and Centro Universitario de la Defensa, Carretera de Huesca s/n, E-50090 Zaragoza, Spain
}

(Received 16 January 2018; revised manuscript received 6 July 2018; published 31 July 2018)

\begin{abstract}
All-optical control of surface plasmon polaritons (SPPs) is theoretically demonstrated in arrays of metallic slits It is shown how the mixing of electromagnetic fields scattered by the slits from a weak beam at $\lambda$ wavelength, with second-harmonic fields generated by a high-intensity $2 \lambda$ beam, creates a destructive interference of surface plasmons in one of the two possible directions of emission from the slits, while these are enhanced along the opposite direction. Our method enables spectrally broadband excitation and thus unidirectional launching of SPP pulses as short as $\sim 20 \mathrm{fs,} \mathrm{all-optically} \mathrm{controlled.} \mathrm{Based} \mathrm{on} \mathrm{these} \mathrm{findings,} \mathrm{an} \mathrm{ultranarrow} \mathrm{bandwidth} \mathrm{surface}$ plasmon frequency comb is designed.
\end{abstract}

DOI: 10.1103/PhysRevB.98.041407

Shortly after the first experimental demonstration of a laser device, Franken et al. reported that the high electromagnetic (EM) fields released by lasers allowed the generation of higher frequencies not present in the laser field [1]. The first nonlinear harmonic discovered was second-harmonic generation (SHG), a process through which two photons with the same frequency generate a new photon with twice the initial frequency. Harmonic generation is behind many of the light technologies developed in the past for both research and commercial purposes [2].

Surface plasmon polaritons (SPPs) are EM modes bound to metal surfaces [3], and it is precisely this confinement which makes them useful for light applications at the nanoscale, mostly developed in the linear regime to date [4,5]. In the last years, nonlinear plasmonics is providing new avenues for the characterization and manipulation of optical signals, sensing, frequency conversion at the nanoscale, and imaging of nano-objects, among others [6]. The new functionalities and applications realized are mainly based on multipolar nonlinear nanoantennas [7-10] or nonlinear optical metasurfaces [11-13], which combine metals with different linear and nonlinear materials.

In this Rapid Communication, we present a method for actively controlling SPPs by means of SHG. We demonstrate the unidirectional emission of SPPs and, based on this, we show dynamical control on the SPP emission. To illustrate this ability we design an ultranarrow bandwidth SPP frequency comb.

We illustrate our approach using a simple structure: a set of slits perforating an opaque metal film. When illuminated from one side by an external light beam, SPP waves are scattered in the opposite side of the film, whenever its electric field oscillates normal to the slit faces. The working principle of our method is depicted in Fig. 1: The SPP fields created by two different sources are coherently interfering on the metal surface at the same wavelength $\lambda$. One source of SPPs are the EM fields

\footnotetext{
*sergut@unizar.es
}

scattered by the slits from a signal beam (central wavelength $\lambda$ ). The second source of SPPs are the second-harmonic ( $\mathrm{SH})$ fields generated in the metallic slits by a control beam (central wavelength $2 \lambda$ ). The EM fields generated at $\lambda$ have different properties of symmetry whether they are linearly generated by the signal beam or originate from SHG. The asymmetries produced in the SPP field distribution can eventually lead to unidirectional emission of SPPs, as shown later.

Other schemes have been reported based on structures similar to the one studied here, where unidirectional launching of SPPs is achieved by means of asymmetric structures $[14,15]$ or illuminating with different polarizations of light holey metal films $[16,17]$ (to name a few).

In general, the optical response of configurations which rely on near-field interactions between different elements forming the system (slits, holes, grooves, ...) are strongly wavelength dependent and the SPP directionality cannot be actively tuned. However, our approach allows us the use of normal incidence illumination, linearly polarized light, and broadband frequency sources (femtosecond lasers), as demonstrated next. In addition, we show that the coherent control of SPPs with SHG is not limited to the investigated structures.

The induced polarization currents and optical near fields are calculated using our homemade finite-difference time-domain (FDTD) code $[18,19]$. The particular details of the nonlinear numerical treatment can be found elsewhere [20] [see also the Supplemental Material (SM) [21]]. Briefly, to calculate the nonlinear response at the SH frequency, the undepleted pump approximation is followed, assuming the intensity of the fundamental-harmonic (FH) field is not affected by the $\mathrm{SH}$ fields. The second-order polarization $\mathbf{P}^{(\mathrm{SH})}$ is introduced as a surfacelike contribution in the FDTD algorithm [22-26],

$$
\begin{aligned}
& \mathbf{P}_{n}^{(\mathrm{SH})}=\left[\chi_{n n n}^{(2)}\left|E_{n}^{(\mathrm{FH})}\right|^{2}+\chi_{n t t}^{(2)}\left|E_{t}^{(\mathrm{FH})}\right|^{2}\right] \mathbf{n}, \\
& \mathbf{P}_{t}^{(\mathrm{SH})}=2 \chi_{t n t}^{(2)} E_{n}^{(\mathrm{FH})} \mathbf{E}_{t}^{(\mathrm{FH})},
\end{aligned}
$$

where $n$ and $t$ stand for normal and tangential to the metal surface, respectively, and $\chi_{i j k}$ are the nonvanishing components of 


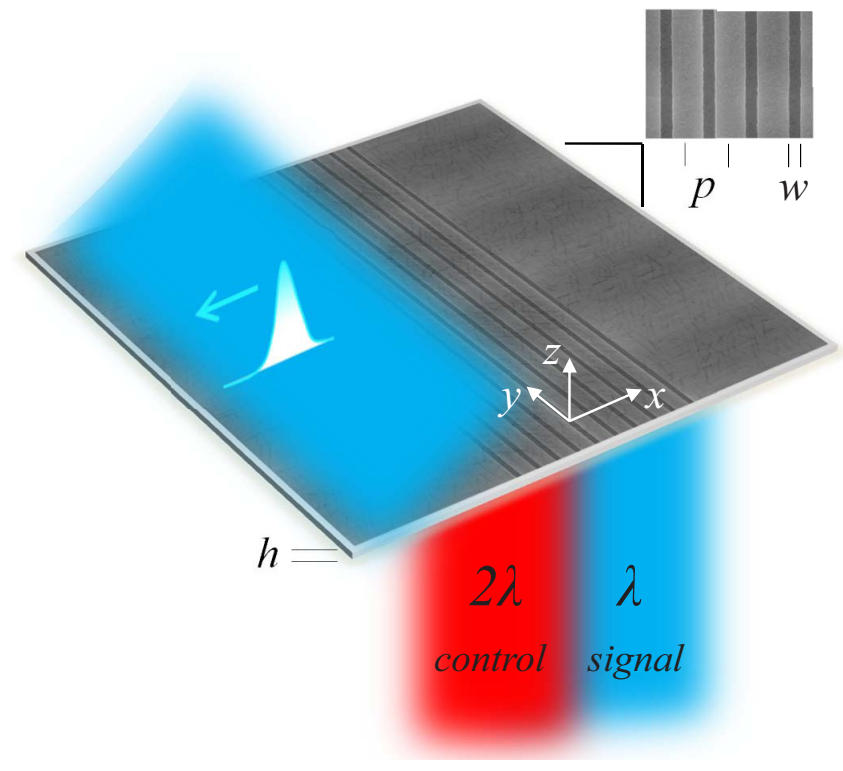

FIG. 1. All-optical control of SPPs by SHG. The simultaneously occurring suppression and enhancement of SPP currents in opposite directions is a consequence of the different symmetry of SPPs generated by a signal beam at $\lambda$ and the $\mathrm{SH}$ field generated by a $2 \lambda$ control beam.

an effective second-order susceptibility tensor, which includes both surface and bulk contributions [25,27,28]. The FH electric field is taken at the metal surface, and from it $\mathbf{P}^{(\mathrm{SH})}$ is calculated at the same location. We have taken the linear dielectric constant of gold and its $\chi^{(2)}$ from Refs. [29,30] and Ref. [27], respectively. We also assumed that $\chi^{(2)}$ is approximately constant for metals in the spectral window of interest [31]. We have checked our FDTD program by comparing numerical against analytical results of SHG efficiency in metal surfaces [32], overall finding good agreement between methods (see Sec. I of the SM [21]).

We are interested in the SPP field distribution at $\lambda$, which depends on the local field generated at SH and the near field scattered by the signal beam. In our proposal only the zeroth diffraction order is allowed at $\lambda$, which ensures the far-field emission at SH is almost suppressed [20]. To do that, we have chosen a finite slit array with period $p=300 \mathrm{~nm}$. In addition, we take the slit width $w=100 \mathrm{~nm}$ and metal thickness $h=$ $300 \mathrm{~nm}$, and the whole system is surrounded by air. The number of slits chosen $(N=17)$ are enough to reproduce the main feature observed in the optical transmission spectrum calculated for the infinite structure [see Fig. 2(a)] [33]. Transmittance is normalized to the amount of light directly illuminating the area of the apertures and the system is illuminated by a plane wave.

In nonlinear simulations the system is illuminated by a low-intensity (real-valued) Gaussian beam at $\lambda=600 \mathrm{~nm}$ (the signal) and by a second high-intensity Gaussian beam at $2 \lambda$ (the control), as illustrated in Fig. 1 [34]. The lateral size ( $x$ direction) of the Gaussian beams is large enough to cover the whole grating [see Fig. 2(d)], and being uniform, the intensity impinging onto the slit array.

Figure 2(b) (top panel) shows a snapshot of the only nonzero component of the magnetic field allowed by symmetry $H_{y}$ when the array is illuminated by a continuous-wave (cw) signal beam (cw illumination is used unless otherwise stated). Superimposed to the color map the white line shows $H_{y}$ at $z=0$. Clearly, the magnetic field has reflection symmetry, that is, $H_{y}$ is an even function regarding the central slit at $x=0\left[H_{y}(-|x|, z)=H_{y}(|x|, z)\right]$ [35]. The field pattern in air demonstrates we succeed in designing the structure because only the zeroth diffraction order is seen in the scattered field of the signal beam. In Fig. 2(b) (bottom panel) the system is solely illuminated by the control beam (signal off). The magnetic field at its SH present point symmetry so $H_{y}$ is an odd function in this case and $H_{y}(-|x|, z)=-H_{y}(|x|, z)$. This is so because centrosymmetric metallic nanostructures have a negligible dipole moment for the illumination chosen [36]. The EM fields at the metal surface "inherit" the symmetry of the EM waveguide modes excited inside the slits, which is different in each case [20]. It is worth mentioning that the waveguide modes excited at SHG are inaccessible by a linearly polarized light for all angles of incidence (see Sec. IV of the SM [21]).

Therefore, by simultaneously switching on the signal and control beams, the final EM field does not have definite symmetry because it results from a sum of odd and even functions. It is precisely the coherent interference of these EM fields which gives rise to unidirectional propagation $[37,38]$. To quantitatively characterize the optical response in terms of the directionality of SPP emission we define the SPP power ratio, for instance, to the right, as $R_{R}=P_{R} /\left(P_{R}+P_{L}\right)$, where $P_{R}$ and $P_{L}$ are the SPP powers to the right and left and calculated on the vertical surfaces represented with dashed lines in Fig. 2(b) (top panel). These surfaces are located far from the slit array and the area of integration has a subwavelength transverse size, so the contribution of radiation from the slits to $P_{R}$ and $P_{L}$ is negligible.

The array of slits is illuminated by both signal and control beams and the results are shown in Figs. 2(c) and 2(d). The interference between the two beams can be tuned by adjusting the relative intensity and/or phase between them, thus $\mathbf{E}_{\text {control }}^{2 \lambda}=\alpha \mathbf{E}_{\text {signal }}^{\lambda}$, where $\alpha=\alpha_{0} \exp (i \delta \pi)$. The parameter $\alpha_{0}$ gives the relative intensity between the control and signal beams and $\delta$ the relative phase between them. In Fig. 2(c) the SPP power ratios to the right and left are shown for different values of the relative phase $\delta$, calculated with FDTD for $\mathrm{cw}$ illumination beams (solid symbols) and for 100-fs laser pulses (open symbols). The trend in both cases is that of a simple sinusoidal function demonstrating the possibility of continuous coherent control of SPPs by SHG (solid and dashed lines). This fact is made more evident in the snapshots of the electric field amplitude calculated for different $\delta$ values and shown in Fig. 2(d) [those labeled with integer numbers and capital letters in Fig. 2(c)]. The top and bottom panels visualize the unidirectional launching of SPPs actively controlled by SHG in each case. Note that all other parameters are kept the same for both light sources (only the pulse duration changes). In the Sec. III of the SM [21], we demonstrate the coherent control of SPPs by SHG with pulses as short as $20 \mathrm{fs}$ (spectral bandwidths of $\approx 370$ and $\approx 90 \mathrm{~nm}$ for the control and signal beams).

The main result of this Rapid Communication is that there exist phase conditions for the total suppression of SPPs in 

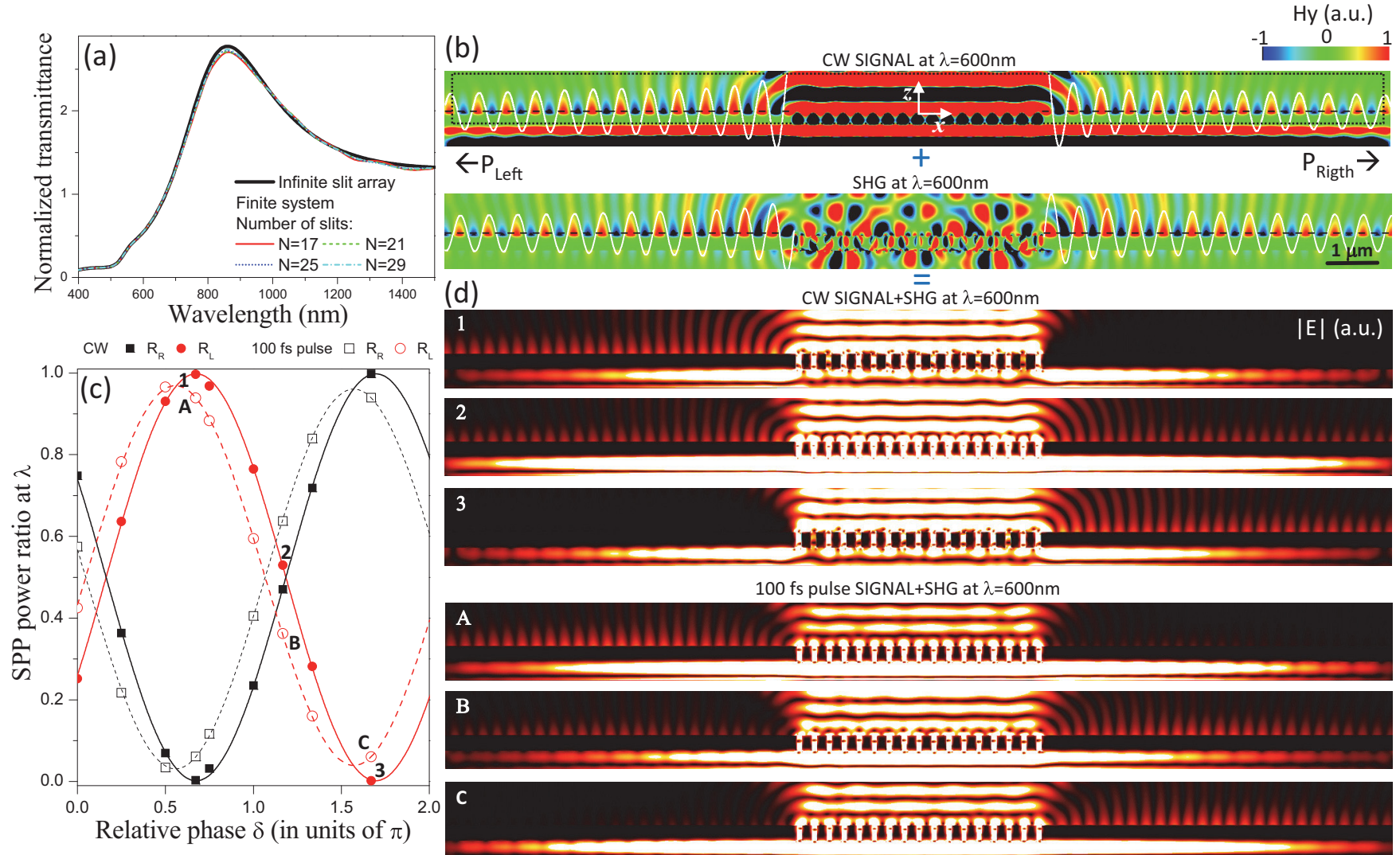

$100 \mathrm{fs}$ pulse SIGNAL+SHG at $\lambda=600 \mathrm{~nm}$

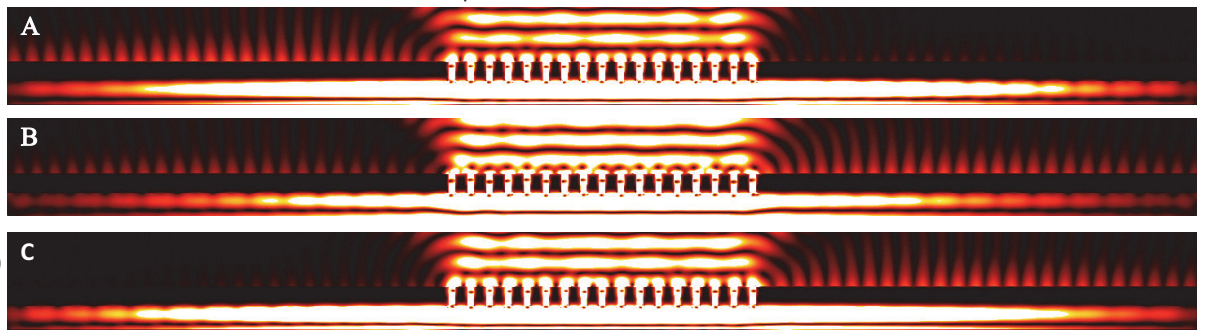

FIG. 2. (a) Linear transmittance (normalized to the amount of light directly illuminating the area of the apertures) through an infinite array of slits (thick line) and different finite systems, for plane-wave illumination. In linear and nonlinear calculations the system is illuminated at normal incidence, and the electric field polarized along the $x$ axis. The geometrical parameters are $p=300 \mathrm{~nm}, w=100 \mathrm{~nm}$, and $h=300 \mathrm{~nm}$. The system is surrounded by air. (b) Top: Real part of the magnetic field component $H_{y}$ scattered from the signal beam at $\lambda=600 \mathrm{~nm}$. Bottom: $H_{y}$ at $\mathrm{SH}$ wavelength generated by a $2 \lambda$ control beam. The white lines show $H_{y}$ at $z=0$. These calculations are obtained with continuous-wave Gaussian beam illumination. (c) Coherent control of SPP directionality by the relative phase $\delta$ between signal and control beams at $\lambda=600 \mathrm{~nm}$. The power ratio to the right (black) and to the left (red) are calculated with FDTD for continuous waves (solid symbols) and for 100-fs pulses (open symbols). The corresponding trends are fitted to sinusoidal functions (solid and dashed lines). (d) Snapshots of the electric field amplitude for the values of $\delta$ labeled with integer numbers and capital letters in (c), and both beams are switched on. Map scale: White (maximum) and black (minimum). (See also the video of 100-fs-laser unidirectional launching SPPs given in the SM).

one direction from the slits, while they are simultaneously enhanced along the opposite direction. The second important message is that our method enables spectrally broadband excitation and thus unidirectional launching and control of SPP wave packets.

For the system and illumination chosen, $\alpha_{0}=83487$. This value corresponds to peak intensities of $I_{c} \approx 0.33 \mathrm{GW} / \mathrm{cm}^{2}$ and $I_{s} \approx 50 \mathrm{~mW} / \mathrm{cm}^{2}$ for the control and signal beams, which are standard in SHG experiments with metallic nanostructures $[39,40]$. These values provide similar SPP field amplitudes on the metal surface through both processes at $\lambda$. Assuming such a condition, a simple relation between the intensities delivered by the signal and control beams can be found, $I_{s}=\beta I_{c}^{2}$, where $\beta$ is a constant. This condition implies that $\alpha_{0}$ is also a function of $I_{c}$, namely, $\alpha_{0}=\sigma / \sqrt{I_{c}}$, where $\sigma$ takes also a constant value. Therefore the parameters $\beta$ and $\sigma$ do not depend on the external light sources, but on the system geometry and the materials through the dielectric constant and $\chi^{(2)}$. Therefore our method is easily scalable in terms of signal and control intensities (see Sec. III in the SM [21]).
In the following, we discuss the possibility to dynamically change the relative phase between the signal and control beams. As a proof of principle, we demonstrate the dynamical control of SPPs by designing a SPP frequency comb. We believe our method is promising to enable the precise control of SPP pulses both in time and frequency domains and further applications must be explored in the future [41,42]. In addition, it is worth mentioning that the SPP frequency comb is generated on site, which is very different from the typical way frequency combs are obtained using mode-locked lasers [43]. To illustrate our method, a single FDTD simulation is set, spanning $T_{\text {simulation }} \sim$ $10 \mathrm{ps}$ in time. The relative phase between the signal and control beams is periodically changed every $T=2 \mathrm{ps}$, producing a SPP left current as shown in Fig. 3(a) with a blue line (EM fields extracted from a point located $9 \mu \mathrm{m}$ from the central slit). The values correspond to an enhancement-suppression sequence in $P_{L}$, in agreement with Figs. 2(c) and 2(d). The Fourier transformation of this train of pulses into the frequency domain produces a SPP frequency comb. The blue curve in Fig. 3(b) shows its spectral response. The frequency comb 

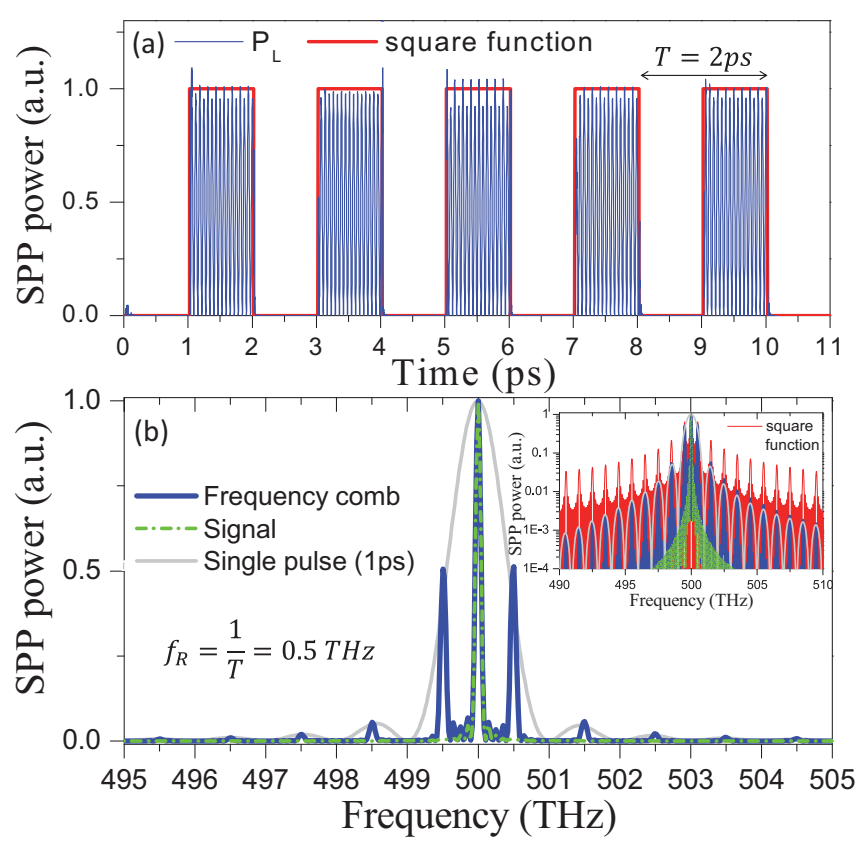

FIG. 3. Demonstration of dynamical control of SPPs at the signal wavelength $\lambda=600 \mathrm{~nm}$. During a single FDTD simulation spanning $T_{\text {simulation }} \sim 10 \mathrm{ps}$, the relative phase between the signal and control beams is periodically changed, producing a train of 1-ps SPP pulses to the left, as shown in (a) with a blue line (EM fields from a point located $9 \mu \mathrm{m}$ to the left from the central slit). These values correspond to an enhancement-suppression sequence in $P_{L}$, in agreement with Fig. 2(c). (b) Demonstration of a SPP frequency comb. The solid blue line shows the spectral response from the EM fields in (a). The frequency comb spacing $f_{R}=1 / T=0.5 \mathrm{THz}$ corresponds to a pulse period $T=2 \mathrm{ps}$. The green dashed line represents the spectral response of the 10-ps signal pulse (control beam switched off) and the gray solid line the corresponding for a single 1-ps pulse. The inset shows the same as that in the main figure but for a wider frequency window (log scale). The spectral response of the square function shown in (a) (red line) is included in the inset.

spacing $f_{R}=0.5 \mathrm{THz}$ corresponds to a pulse period $T=2$ ps. The spectral response of the 10-ps signal pulse (control beam switched off) and the corresponding for a 1-ps single pulse are shown with a green dashed line and a gray solid line, respectively. The inset shows the same but for a wider frequency window. In the inset, the spectral response of the square function represented in Fig. 3(a) with a red line shows the same spectral pattern of the SPP frequency comb, as expected.

Regarding the experimental feasibility of our proposal, our ideas can be experimentally verified using pump-probe spectroscopy. The light sources involved have different frequencies and their phases need to be locked, as in experiments for terahertz wave generation [44]. In these experiments the control beam is focused onto a SHG crystal to generate enough output (signal) with a fixed phase relation between them. In addition, the fine control of the relative phase required for the coherent control of SPPs by SHG, especially when using fs pulses, could be achieved using a fused silica wedge pair (small angle) to finely control the delay and so the relative phase between signal and control beams. Attosecond phasecontrol accuracy has been reported using this kind of phase compensator [45], a short enough time resolution to cover a complete relative phase change of $\pi$ in our notation, which means going from point 1 to 3 in Fig. 2(d).

The pulse duration and the switching rate of the phase delay in the frequency comb simulations are 10 and $2 \mathrm{ps,}$ respectively. These values have been chosen to reduce the computational burden, thus other pump-probe configurations are possible from picosecond to $\mathrm{cw}$ operating lasers. Therefore our proposal should be also scalable in terms of pulse duration. We think the best way to experimentally achieve such high switching rates of the phase is by acting on the optical path of the laser beams with the technique explained above [45].

The use of SHG for coherently controlling radiation has been recently suggested using isolated metallic wires [46]. Several drawbacks are inherent in the study of SHG from single nanoparticles, but even for flat metal surfaces there is still some controversy given the subtle nature of SHG processes in metals $[28,47,48]$. Even though most of the basic research on SHG from metals was initially focused on metal nanoparticles $[7,28,49,50]$ (to name a few), note that only recently the optical characterization of SHG emission from a single nanoparticle has been experimentally attained [51]. Complex setups are needed to investigate far-field SHG from single metallic nanoparticles as compared to extended systems such as arrays of symmetric nanoparticles [52] or holey metal films [53].

Our approach to all-optical control of SPPs based on SHG is feasible in metallic structures supporting different plasmonic modes. As an example, unidirectional emission of long-range SPPs [54] is demonstrated with a single metallic nanowire located over an optically thin metal film (akin to patch antennas [55]) in Sec. V of the SM [21].

In addition to the active control of confined modes such as SPPs being attainable using our method, the coherent control of radiation from the corrugated metal surface is also feasible, as shown in Sec. II of the SM [21].

In conclusion, we have theoretically demonstrated the possibility of building spectrally broadband unidirectional SPP sources with pulses as short as $\sim 20 \mathrm{fs}$ and actively controlled by an external beam. Our theoretical proposal can be experimentally verified using standard pump-probe spectroscopy. We envisage nonlinear nanodevices for the control and generation of light based on coherently combining multipolar modes excited by SHG. As a proof of principle of such a nanodevice, we have designed a SPP frequency comb following our method. Our proposal is of a general application and would work in different plasmonic platforms and frequency regimes.

We acknowledge support from the Spanish Ministry of Science, Innovation and Universities under Project No. MAT2017-88358-C3-2-R (AEI/FEDER,UE). 
[1] P. A. Franken, A. E. Hill, C. W. Peters, and G. Weinreich, Phys. Rev. Lett. 7, 118 (1961).

[2] E. Garmire, Opt. Express 21, 30532 (2013).

[3] W. Barnes, A. Dereux, and T. Ebbesen, Nature (London) 424, 824 (2003).

[4] L. Novotny and B. Hecht, Principles of Nano-Optics (Cambridge University Press, Cambridge, UK, 2012).

[5] A. I. Fernández-Domínguez, F. J. García-Vidal, and L. MartínMoreno, Nat. Photonics 11, 8 (2017).

[6] M. Kauranen and A. V. Zayats, Nat. Photonics 6, 737 (2012).

[7] G. Bautista, M. J. Huttunen, J. Mäkitalo, J. M. Kontio, J. Simonen, and M. Kauranen, Nano Lett. 12, 3207 (2012).

[8] J. Butet, I. Russier-Antoine, C. Jonin, N. Lascoux, E. Benichou, and P.-F. Brevet, Nano Lett. 12, 1697 (2012).

[9] H. Harutyunyan, G. Volpe, and L. Novotny, in Optical Antennas, edited by M. Agio and A. Alù (Cambridge University Press, Cambridge, UK, 2012), Chap. 8.

[10] D. Smirnova and Y. S. Kivshar, Optica 3, 1241 (2016).

[11] J. Lee, M. Tymchenko, C. Argyropoulos, P.-Y. Chen, F. Lu, F. Demmerle, G. Boehm, M.-C. Amann, A. Alú, and M. A. Belkin, Nature (London) 511, 65 (2014).

[12] A. E. Minovich, A. E. Miroshnichenko, A. Y. Bykov, T. V. Murzina, D. N. Neshev, and Y. S. Kivshar, Laser Photonics Rev. 9 (2015).

[13] F. Wang, A. B. F. Martinson, and H. Harutyunyan, ACS Photonics 4, 1188 (2017).

[14] F. Lopez-Tejeira, S. G. Rodrigo, L. Martin-Moreno, F. J. GarciaVidal, E. Devaux, T. W. Ebbesen, J. R. Krenn, I. P. Radko, S. I. Bozhevolnyi, M. U. Gonzalez et al., Nat. Phys. 3, 324 (2007).

[15] X.-Y. Song, Z. Zhang, H. Liao, Z. Li, C. Sun, J. Chen, and Q. Gong, Nanoscale 8, 6777 (2016).

[16] F. J. Rodríguez-Fortuño, G. Marino, P. Ginzburg, D. O’Connor, A. Martínez, G. A. Wurtz, and A. V. Zayats, Science 340, 328 (2013).

[17] J. Lin, J. P. B. Mueller, Q. Wang, G. Yuan, N. Antoniou, X.-C. Yuan, and F. Capasso, Science 340, 331 (2013).

[18] A. Taflove and S. C. Hagness, Computational Electrodynamics: The Finite-Difference Time-Domain Method, 3rd ed. (Artech House, Boston, 2005).

[19] S. G. Rodrigo, Optical Properties of Nanostructured Metallic Systems: Studied with the Finite-Difference Time-Domain Method, Springer Theses (Springer, Berlin, 2011).

[20] S. G. Rodrigo, V. Laliena, and L. Martín-Moreno, J. Opt. Soc. Am. B 32, 15 (2015).

[21] See Supplemental Material at http://link.aps.org/supplemental/ 10.1103/PhysRevB.98.041407 for Sec. I shows a comparison between FDTD calculations against analytical results of SHG emission from a flat metal surface; Sec. II shows coherent control of lattice diffraction orders by SHG; Sec. III presents a study about how the coherent control of SPPs by SHG depends on the pump-probe configuration; Sec. IV is a study of the symmetry properties of SHG fields in a single metallic slit; Sec. V demonstrates coherent control of long-range SPPs.

[22] N. Bloembergen, R. K. Chang, S. S. Jha, and C. H. Lee, Phys. Rev. 174, 813 (1968).
[23] J. E. Sipe, V. C. Y. So, M. Fukui, and G. I. Stegeman, Phys. Rev. B 21, 4389 (1980).

[24] M. Corvi and W. L. Schaich, Phys. Rev. B 33, 3688 (1986).

[25] P. Guyot-Sionnest and Y. R. Shen, Phys. Rev. B 38, 7985 (1988).

[26] V. Mizrahi and J. E. Sipe, J. Opt. Soc. Am. B 5, 660 (1988).

[27] F. X. Wang, F. J. Rodríguez, W. M. Albers, R. Ahorinta, J. E. Sipe, and M. Kauranen, Phys. Rev. B 80, 233402 (2009).

[28] C. Ciracì, E. Poutrina, M. Scalora, and D. R. Smith, Phys. Rev. B 86, 115451 (2012).

[29] F. Hao and P. Nordlander, Chem. Phys. Lett. 446, 115 (2007).

[30] S. G. Rodrigo, F. J. García-Vidal, and L. Martín-Moreno, Phys. Rev. B 77, 075401 (2008).

[31] D. Krause, C. W. Teplin, and C. T. Rogers, J. Appl. Phys. 96, 3626 (2004).

[32] K. A. O’Donnell and R. Torre, New J. Phys. 7, 154 (2005).

[33] A. I. Fernández-Domínguez, F. J. García-Vidal, and L. MartínMoreno, Phys. Rev. B 76, 235430 (2007).

[34] S. G. Rodrigo, S. Carretero-Palacios, F. J. García-Vidal, and L. Martín-Moreno, Phys. Rev. B 83, 235425 (2011).

[35] A. Y. Nikitin, S. G. Rodrigo, F. J. García-Vidal, and L. MartínMoreno, New J. Phys. 11, 123020 (2009).

[36] M. Finazzi, P. Biagioni, M. Celebrano, and L. Duò, Phys. Rev. B 76, 125414 (2007).

[37] M. J. Huttunen, J. Mäkitalo, G. Bautista, and M. Kauranen, New J. Phys. 14, 113005 (2012).

[38] I. M. Hancu, A. G. Curto, M. Castro-López, M. Kuttge, and N. F. van Hulst, Nano Lett. 14, 166 (2014).

[39] M. E. Inchaussandague, M. L. Gigli, K. A. O’Donnell, E. R. Méndez, R. Torre, and C. I. Valencia, J. Opt. Soc. Am. B 34, 27 (2017).

[40] F. Wang and H. Harutyunyan, Opt. Express 26, 120 (2018).

[41] S. T. Cundiff and J. Ye, Rev. Mod. Phys. 75, 325 (2003).

[42] Nat. Photonics 5, 185 (2011).

[43] A. Bartels, D. Heinecke, and S. A Diddams, Science 326, 681 (2009).

[44] J. Liu, J. Dai, S. L. Chin, and X.-C. Zhang, Nat. Photonics 4, 627 (2010).

[45] J. Dai and X.-C. Zhang, Appl. Phys. Lett. 94, 021117 (2009).

[46] S. G. Rodrigo, H. Harutyunyan, and L. Novotny, Phys. Rev. Lett. 110, 177405 (2013).

[47] N. B. Grosse, J. Heckmann, and U. Woggon, Phys. Rev. Lett. 108, 136802 (2012).

[48] K. N. Reddy, P. Y. Chen, A. I. Fernández-Domínguez, and Y. Sivan, J. Opt. Soc. Am. B 34, 1824 (2017).

[49] G. S. Agarwal and S. S. Jha, Solid State Commun. 41, 499 (1982).

[50] J. I. Dadap, J. Shan, K. B. Eisenthal, and T. F. Heinz, Phys. Rev. Lett. 83, 4045 (1999).

[51] J. Butet, J. Duboisset, G. Bachelier, I. Russier-Antoine, E. Benichou, C. Jonin, and P.-F. Brevet, Nano Lett. 10, 1717 (2010).

[52] M. D. McMahon, R. Lopez, R. F. Haglund, E. A. Ray, and P. H. Bunton, Phys. Rev. B 73, 041401 (2006).

[53] J. A. H. van Nieuwstadt, M. Sandtke, R. H. Harmsen, F. B. Segerink, J. C. Prangsma, S. Enoch, and L. Kuipers, Phys. Rev. Lett. 97, 146102 (2006).

[54] E. N. Economou, Phys. Rev. 182, 539 (1969).

[55] M. Manjare, F. Wang, S. G. Rodrigo, and H. Harutyunyan, Appl. Phys. Lett. 111, 221106 (2017). 